\title{
CRISIS ECONOMICA Y ESTABILIDAD BANCARIA EN EL PERU 2007 - 2015
}

\author{
ECONOMIC CRISIS AND BANK STABILITY IN PERU 2007 - 2015
}

\author{
${ }^{1}$ Juan Magallanes Díaz
}

\begin{abstract}
Resumen
La inestabilidad y crisis económica es inherente a toda economía de mercado, y esta jala a todos los sectores, incluido al bancario. A pesar de estos avatares, este último tiene que mostrar un mínimo de solidez y estabilidad del sistema para mantener la confianza del mercado. El presente documento tiene como propósito analizar la estabilidad de la banca múltiple y comercial en el Perú en los últimos nueve años (2007-2015). Para ello, se utiliza un sistema de calificación para 13 indicadores financieros de los principales bancos del país que representan más del 85\% de las colocaciones, de acuerdo a su comportamiento histórico o distribución de probabilidad. Se concluye y demuestra que el sistema bancario está dando muestra de estar operando en riesgo moderado y de estabilidad bancaria, con tendencia a consolidar esta posición.
\end{abstract}

Palabras claves: estabilidad bancaria, riesgo bancario, crisis económica.

\begin{abstract}
Instability and economic crisis is inherent in any market economy, and this pulls all sectors, including banking. Despite these burdens, the latter must show a minimum of solidity and stability of the system to maintain the market in confidence. This paper aims to analyze the stability of multiple and commercial banks in Peru in the last nine years (2007-2015). For this purpose, a rating system is used for 13 financial indicators of major banks representing more than $85 \%$ of the loans, according to their historical behavior or probability distribution. It concludes and demonstrates that the banking system is showing signs of operating in moderate risk and banking stability, tending to consolidate this position.
\end{abstract}

Keywords: banking stability, bank risk, economic crisis.

\section{Introducción}

El logro de un crecimiento económico sólido debe estar acompañado de un sistema bancario de la misma característica, que responda y acompañe financiando la expansión de la inversión, y mostrando también solidez y estabilidad en los momentos difíciles de crisis, paso obligado de toda economía capitalista. Los primeros 15 años del presente siglo la economía nacional y mundial han crecido rápido, pero también han pasado por fuertes caídas y de recesión de la que aún no se sale.

Las grandes empresas de inversiones, los gobiernos, las entidades supervisoras y los propios bancos han mostrado interés por demostrar que se tiene una buena posición de estabilidad bancaria para transmitir confianza a los mercados. Las interrupciones en el buen funcionamiento de la industria bancaria tienden a exacerbar las fluctuaciones producto. En consecuencia, las crisis bancarias se asocian en forma significativa con las pérdidas de producción económica. De ello se desprende que la preservación de la estabilidad en el sector bancario es de suma importancia para los supervisores bancarios (De Jonghe 2010). Muchos autores proporcionan una mayor evidencia que el grado de desarrollo del sector financiero actúa como un contribuyente importante al crecimiento económico (Levine 1997; Levine 1998; Levine, Loayza, y Beck 2000; King y Levine 1993b; King y Levine 1993a; Demirgüç-Kunt y Maksimovic 1998; Rajan y Zingales 1998).

El presente estudio tiene como objetivo demostrar si es que el sistema bancario en el Perú tiene una posición sólida comprobada en indicadores de estabilidad bancaria donde se constate un nivel de riesgo bajo en presencia de desaceleración y/o crisis económica o de shocks externos, y en su tendencia en el corto y mediano plazo. Para ello se han revisado varios modelos, de los cuales el utilizado aquí responde a criterios de calificación de un conjunto de variables financieras de los bancos seleccionados y de la respectiva distribución estadística de sus datos históricos.

\section{Revisión de literatura}

Existe mucho interés por parte de las autoridades monetarias y financieras en conocer algún indicador que muestre el grado de solidez o debilidad que tiene el sistema financiero, esto debido a las crisis ocurridas

\footnotetext{
${ }^{1}$ Economista, Mg. Sc. en Economía Agrícola y Desarrollo Rural, profesor principal del Departamento Académico de Economía y Planificación de la Universidad Nacional Agraria La Molina, Lima, Perú. E-mail: jmagallanes@lamolina.edu.pe

La investigación contó con la asistencia del egresado en economía José Huerta López.
} 
en las dos últimas décadas y sobretodo la del 2007 2008 más conocida como "Gran Depresión", y al alto costo económico y social que generan los periodos de inestabilidad financiera (Delgado y García 2013). Sin embargo, este hecho es un reto pues no se ha llegado siquiera a un consenso en la definición de crisis financiera. Para poder estimar algún indicador de estabilidad financiera, se han realizado trabajos bajo el enfoque de alerta temprana o Early Warning System (EWS) con la finalidad de prevenir crisis financieras futuras. Estos modelos están diseñados para identificar situaciones de estrés de manera anticipada, usando una ecuación reducida, en donde la crisis es una variable dicotómica (0 y 1) donde 1 es presencia de crisis (Borio y Drehmann 2009)"mendeley" : \{ "formattedCitation" : "(Borio y Drehmann 2009. Sin embargo, Estrada y Morales (2009) afirman que ello no debería ser representado de manera binaria pues un escenario de estrés financiero es un estado continuo, y este describe el sector en cada periodo de tiempo produciendo una foto actual del nivel de estrés contemporáneo que presenta el sistema. Además, Jokipii y Monnin (2013) argumenta que los autores se han centrado en estudiar los impactos en la economía real durante o después de periodos de crisis debido a que han elegido trabajar con variables dependientes binarias, por lo que se ha ignorado ese impacto durante periodos más estables.

El Fondo Monetario Internacional (IMF 2006) dada su función de apoyar a mantener una salud financiera brinda su juicio experto de indicadores de solidez financiera, para auxiliar a los distintos bancos centrales, que puedan usar con la finalidad que puedan hacer prevalecer su estabilidad.

Tomando como referencia estos indicadores, el Banco Central de la República Dominicana publicó un documento de trabajo sobre un índice de estabilidad bancaria para el mismo país (Delgado y García 2013). Considerando las recomendaciones de Indicadores de Solidez Financiera del FMI y aplicando la metodología de componentes principales, se utilizaron los balances financieros de los bancos para desarrollar índices de estabilidad para distintos subsectores bancarios como lo son los bancos múltiples, asociaciones de ahorro y préstamo, y bancos de ahorro y crédito; los resultados sugieren que los índices tienen capacidad de capturar los períodos de estrés experimentados por el sector bancario, reaccionando especialmente a la crisis bancaria 2003-2004 vividas en ese país, así como a otros hechos relevantes en ese período; además que los índices de estabilidad bancaria obtenidos se encuentran cointegrados con variables del entorno macroeconómico.

Bajo esta misma perspectiva, Estrada y Morales (2009) desarrollaron un índice de estabilidad financiera para Colombia la cual no solo es continúa sino también puede tomar valores positivos y negativos, donde si el índice es mayor a cero entonces significa un nivel de estrés financiero mayor al promedio y si es negativo, mayor estabilidad que el promedio. A diferencia del resto, este autor desarrolla indicadores de estrés por tipo de entidad bancaria: banca comercial, banca especializada en crédito hipotecario, compañías de financiamiento comercial y cooperativas financieras. Los métodos usados son el de igualdad de varianzas, componentes principales, y regresiones Zero-Inflated Poison (ZIP) y Zero-Inflated Binomial Negative (ZIBN).

Para el caso de Canadá, Illing y Liu (2003) desarrollaron un índice continuo de estrés financiero incluyendo datos del sector bancario, tipo de cambio, deuda y mercado de capitales, el cual es construido usando técnicas como análisis de factores, análisis de corte transversal y modelos GARCH.

Por otro lado, Puddu (2008) desarrolló índices de estrés bancario basados en dos enfoques: el de señales y el de estimaciones de modelos Zero-Inflated Poisson (ZIP). El autor encuentra que estas metodologías brindan mejores resultados que las de igualdad de varianza y análisis de factores.

No todos los autores toman rigurosamente en cuenta las recomendaciones hechas por el FMI, por su lado Jahn y Kick ( $\sin$ fecha) desarrolla un continuo y prospectivo indicador de estabilidad para el sistema bancario. Aplica una la técnica de regresión panel e identifica un indicador macroprudencial de alerta temprana. Encuentra que el precio de activos, indicadores del ciclo de negocio e indicadores del mercado de dinero son indicadores de alerta temprana relevantes. Además, afirman que el ratio crédito-PBI son más importantes para bancos cooperativos y menos relevantes para los bancos comerciales.

De modo similar, Ortiz y Ugarte (2015) realizan un indicador de crisis bancaria utilizando un modelo no lineal de datos panel. Este tiene como variable principal el ratio crédito sobre PBI. Con el objetivo de desarrollar un índice con el más alto desempeño predictivo desarrollan su propia brecha de crédito la cual está en función del ratio crédito-PBI y el ingreso per cápita. Este indicador es comparado con otros indicadores de exceso de crédito usado por instituciones internacionales, luego este indicador junto con otros es seleccionado mediante el Modelo Bayesiano Promedio (BMA por sus siglas en inglés) para formar un modelo multivariado con el que se construirá el modelo de alerta temprana.

Jokipii y Monnin (2013) miden la estabilidad del sector bancario basado en la probabilidad de default del sector bancario, es decir, si al final del periodo los activos no son suficientes para pagar las deudas. Encuentran que la estabilidad del sector bancario es un conductor importante del crecimiento del PBI futuro y que la inestabilidad del sector es seguida por la incertidumbre del crecimiento del producto. 


\section{Materiales y métodos}

Se proponen las hipótesis siguientes; primero, de que el sistema bancario representado en sus principales bancos comerciales están demostrando tener una estabilidad bancaria y por lo tanto de baja exposición al riesgo; segundo, que a pesar de las vicisitudes económicas la tendencia es hacia un mejor nivel de estabilidad bancaria en el mediano plazo.

Para el propósito del presente trabajo se utilizará la metodología VaR (Value at Risk). Esta metodología, propuesta por la teoría de administración de riesgo, considera escenarios de estrés permite estimar el valor máximo que puede perder una acción o portafolio dado un periodo de tiempo y un intervalo de confianza (Espino 2012). A lo que se refiere este intervalo es que

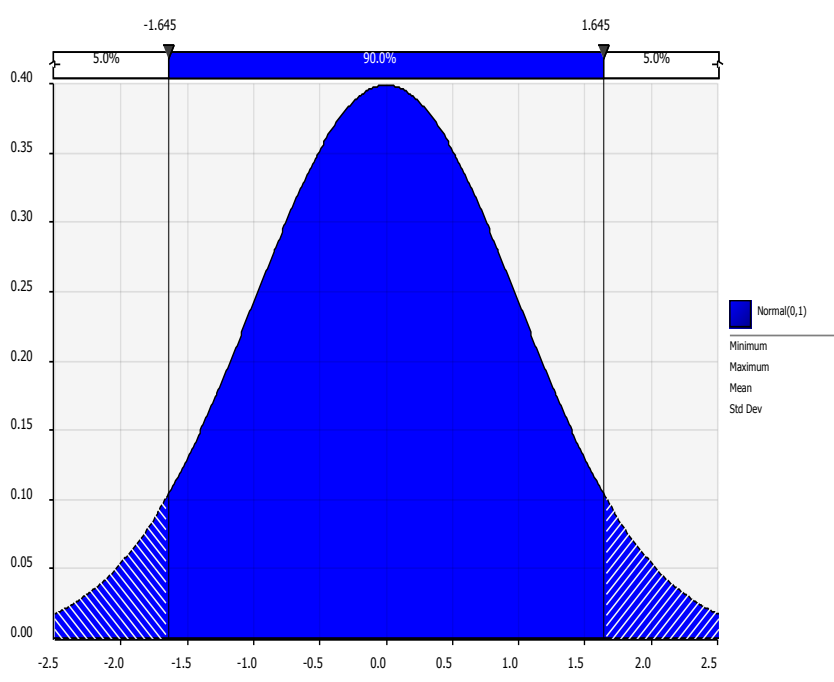

Gráfico 1. Función de distribución de probabilidad.

Para este trabajo el uso de metodología VaR toma en cuenta un escenario de estrés con un $90 \%$ de intervalo de confianza, es decir, en $10 \%$ de los casos ocurrirá una situación de estrés. Una vez definido el valor estimado de pérdida, se divide la distribución en 6 intervalos qué serán útiles para asignar un puntaje a cada indicador. Para esto se toman valores del percentil 10, 20, 50, 80 y 90 de cada indicador. Posteriormente se asignarán valores del 1 al 6 , "normalizados", a cada intervalo con el fin de ver los cambios en los niveles de riesgo. Las variables e indicadores a considerar, 13 en total, son presentados en ratios financieros, específicamente, los relacionados a la posición del capital, a la calidad de la cartera de colocaciones, a los gastos administrativos, a la rentabilidad, al grado de liquidez, al grado de dolarización de las colocaciones, a la interconexión con el sistema bancario y a la exposición externa, todos ellos recomendados por el IMF (2006). Se identifica un escenario de estrés para cada uno de los ratios por cada banco, de esa manera se obtendrá un Índice de Estabilidad Bancaria (IEB). si, por ejemplo, fuera de $95 \%$, entonces si hubiera 100 escenarios de estrés, en 5 de ellos se obtendría la pérdida máxima. Esto es equivalente a estimar el percentil 5 de una distribución a partir del cual se estima la pérdida máxima con una probabilidad de $5 \%$.

Si se considera de $95 \%$ el nivel de confianza, el valor en riesgo (VaR) de la variable a analizar sería el valor que se obtiene en el 5\% del área de la función de distribución de probabilidad, para esto se trabaja y obtiene la función respectiva que más se ajuste a los datos de cada una de los trece indicadores que serán trabajadas en el Índice de Estabilidad Bancaria, y no necesariamente se considerara como única la distribución Normal de la data histórica, tal como lo recomienda el mismo Espino (2012). Así también, para la obtención de estas funciones se hizo uso del software estadístico del@risk.

Tabla 1. Indicadores Financieros para la Estimación del Índice.

\begin{tabular}{|l|l|}
\hline \multicolumn{1}{|c|}{ Indicadores / Percentíl } & \\
\hline Capital (C) & - \\
1 Pasivo / Patrimonio & - \\
2 (Cartera Morosa - Provisiones) / Patrimonio & - \\
\hline Calidad de Cartera (A) & - \\
3 Cartera Morosa / Colocaciones Brutas & \\
4 Bienes Adjudicados / Activos Totales & - \\
\hline Administración (M) & - \\
5 Gastos Administrativos / Activos Totales & \\
6 Gastos Adm./Margen Financ. Ingresos No & + \\
\hline Fentabianc.) & + \\
7 Utilidad Anual / Activo (ROA) & \\
\hline Liquidez (L) & - \\
8 Fondos Disponibles / Depósitos Totales & - \\
9 Fondos Disponibles / Depósitos Vista & - \\
\hline Dolarización (D) & - \\
10 Colocaciones ME / Colocaciones Totales \\
11 Depósito ME / Depósitos Totales & - \\
\hline Interconexión (I) & \\
12 Préstamos Interbancarios / Patrimonio & \\
\hline Exposición Externa (EE) & \\
13 Adeudados Externos / Activos & \\
\hline
\end{tabular}

La muestra está compuesta por los cuatro bancos más grandes del Perú teniendo en cuenta su participación en el total activos de la banca múltiple ya que juntos representan más del $85 \%$. Los valores de referencia son los ratios del total de la banca múltiple durante el periodo 2007-2015 y como los datos están organizados de manera mensual, nos da como resultado una serie de 96 observaciones ( 8 años) por indicador, cuya fuente de datos es la consignada en la Superintendencia de Banca, Seguros y AFP del Perú.

Los valores de referencia para cada ratio se escogen en niveles y en diferencia. En otras palabras, se usará el valor del ratio en valor absoluto (niveles), pero también se hará uso de su variación respecto del periodo anterior (diferencia). Un ratio de pasivo/patrimonio de $10 \%$ se 
encuentra en niveles; sin embargo, también es importante evaluar la diferencia en tanto nos muestra su tendencia, por ejemplo, el ratio cartera morosa/colocaciones brutas pasó de $10 \%$ a $12 \%$ en un año. De esta manera, el IEB estará compuesto por dos indicadores: en niveles y en diferencia.

Se asigna puntajes del 1 al 6 a cada ratio financiero por cada banco donde valores cercanos a 1 significa mayor estabilidad y cercano a 6, menor estabilidad. Algunos ratios financieros tienen una relación positiva con la estabilidad y otros, una relación negativa. En tanto tenga una relación negativa, se dará un puntaje de 6 al ratio cuando pase del percentil 90. De manera contraria, cuando el ratio tenga relación positiva, entonces se asignará un puntaje de 1 cuando este pase del percentil 90.

Con estos datos se hace un promedio simple por banco de los puntajes asignados a ellos.

Donde:

$$
I E B_{i}=\frac{\sum_{j}^{M} \beta_{j}}{M}
$$

El último paso es la agregación del sistema bancario, usando el IEB de cada banco, mediante un promedio ponderado. En tanto la dificultad de establecer las ponderaciones de las variables yace en la falta de un indicador de referencia que permita verificar la precisión de los pesos y hacer pruebas con estos (Estrada y Morales 2009), en este caso, el factor de ponderación es la participación i en el total de activos del sistema en cada periodo.

Donde:

$$
I E B=\sum_{1}^{N} \alpha_{i} \times I E B_{i}
$$

\section{Resultados}

\subsection{Selección de la banca referencial}

Se procedió a obtener el indicador de estabilidad bancaria referencial o benchmarking, sumando la desviación estándar de la variación porcentual mensual de las colocaciones, de los depósitos y de las utilidades netas de cada uno de los cuatro bancos grandes y del promedio de la banca múltiple, lo que se presenta en la tabla 2; por razones de prudencia se omite en este cuadro el nombre de los bancos respectivos. El resultado para la el total de la banca múltiple fue de $25.86 \%$, muy alineado a uno de los bancos con gran respaldo internacional. Uno de ellos mostró una mayor volatilidad debido a variaciones muy marcadas en la variable de utilidad neta en el horizonte del estudio. Se decidió considerar por ello al total de la Banca Múltiple como el referencial.

Tabla 2. Selección de Bancos

\begin{tabular}{|c|c|}
\hline Banco 1 & $24.74 \%$ \\
\hline Banco 2 & $34.39 \%$ \\
\hline Banco 3 & $39.08 \%$ \\
\hline Banco 4 & $136.61 \%$ \\
\hline Total Banca Multiple & $\mathbf{2 5 . 8 6 \%}$ \\
\hline
\end{tabular}

Fuente: Estadísticas SBS 2007-2015

Seguidamente se procedió a estimar los valores de referencia de estabilidad bancaria de cada uno de los 13 indicadores financieros obtenido del total de la Banca Múltiple que funciona en nuestro mercado, en niveles y en diferencia (últimos doce meses) durante el período 2007 2015. Las tablas 3 y 4 contiene los resultados de ello, y los cálculos de los ratios y sus respectivos histogramas, así como la línea de la función de distribución que más se ajusta a los datos, son presentados en el anexo; las funciones de distribución loglogist y logist fueron las obtenidas con más frecuencia.

Un ejemplo de los cálculos realizados para los 13 indicadores es el que se ilustra en el gráfico 2, donde se trabajó el indicador Cartera morosa / Colocaciones Brutas, y con el apoyo del software@ @risk nos resultó la distribución loglogist como la modelación de función de distribución de probabilidad que mejor se ajusta a los datos observados (108 meses en total). Considerando un análisis de estrés, al 10\% de nivel de confianza tenemos un ratio de Valor en riesgo equivalente a 1.418 de porcentaje de cartera morosa, y así se sigue estimando al $20 \%$, al $50 \%, 80 \%$ y $90 \%$ respectivamente.

Tomando en cuenta el tipo de relación entre el ratio Gráfico 2. Fit Comparison for Cartera Morosa/Colocaciones Brutas.

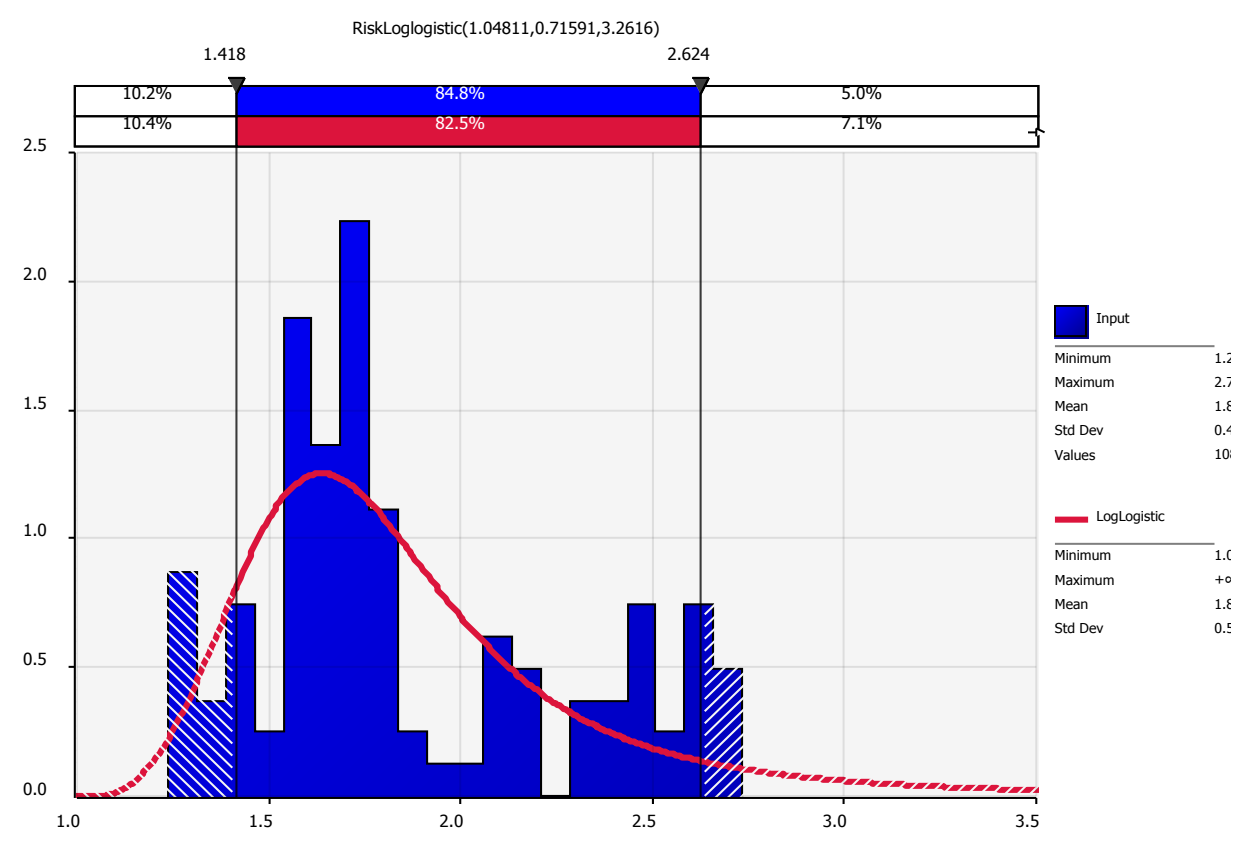


financiero de cada banco con el de estabilidad bancaria obtenido y presentados en las tablas 3 y 4 , se procedió a calificar dicha relación. A aquellos ratios que tienen una relación negativa, se le ha otorgado un puntaje de 6 cuando pasan el percentil 90, y si tienen una relación positiva se le da el puntaje de 1 (uno). Por ejemplo, en el caso de apalancamiento (pasivo / patrimonio), cuando el indicador de un banco sea mayor a $10.38 \%$ se le otorgará el puntaje de 6. En el caso del ROA, cuando el ratio refleje una mayor rentabilidad, que supere supere el $2.66 \%$ se le asignará el puntaje de 1 .

Tabla 4. Percentiles por indicador (en porcentaje)

Indicadores expresados en Diferencia.

\begin{tabular}{|c|c|c|c|c|c|c|c|}
\hline Indicadores / Percentíl & & 10 & 20 & 50 & 80 & 90 & F. Distribución \\
\hline \multicolumn{8}{|l|}{ Capital (C) } \\
\hline 1 Pasivo / Patrimonio & - & -0.3707 & -0.2569 & -0.0417 & 0.2028 & 0.3609 & Logistic \\
\hline 2 (Cartera Morosa - Provisiones) / Patrimonio & - & -1.3059 & -0.8453 & -0.0578 & 0.7296 & 1.1903 & Logistic \\
\hline \multicolumn{8}{|l|}{ Calidad de Cartera (A) } \\
\hline 3 Cartera Morosa / Colocaciones Brutas & - & -0.0738 & -0.0457 & 0.0084 & 0.0602 & 0.0858 & Weibull \\
\hline 4 Bienes Adjudicados / Activos Totales & - & -0.0171 & -0.0109 & -0.0005 & 0.0099 & 0.0160 & Logistic \\
\hline \multicolumn{8}{|l|}{ Adminis tración (M) } \\
\hline 5 Gastos Administrativos / Activos Totales & - & -0.1317 & -0.0870 & -0.0105 & 0.0660 & 0.1107 & Logistic \\
\hline 6 Gastos Adm./(Margen Financ.+Ingresos No Financ.) & - & -6.6865 & -4.3663 & 0.0724 & 4.5111 & 6.8313 & Normal \\
\hline \multicolumn{8}{|l|}{ Rentabilidad (E) } \\
\hline 7 Utilidad Anual / Activo (ROA) & + & -0.1449 & -0.0927 & -0.0034 & 0.0858 & 0.1381 & Logistic \\
\hline \multicolumn{8}{|l|}{ Liquidez (L) } \\
\hline 8 Fondos Disponibles / Depósitos Totales & + & -2.6704 & -1.6881 & -0.0089 & 1.6703 & 2.6526 & Logistic \\
\hline 9 Fondos Disponibles / Depósitos Vista & + & -12.1849 & -7.7814 & -0.2535 & 7.2744 & 11.6779 & Logistic \\
\hline \multicolumn{8}{|l|}{ Dolarización (D) } \\
\hline 10 Colocaciones ME / Colocaciones Totales & - & -0.9297 & -0.6817 & -0.2578 & 0.1661 & 0.4141 & Logistic \\
\hline 11 Depósito ME / Depósitos Totales & - & -9.2805 & -6.1282 & -0.0807 & 5.9890 & 9.1706 & Gamma \\
\hline \multicolumn{8}{|l|}{ Interconexión (I) } \\
\hline 12 Préstamos Interbancarios / Patrimonio & - & -0.5161 & -0.3287 & -0.0049 & 0.3231 & 0.5170 & LogLogistic \\
\hline \multicolumn{8}{|l|}{ Exposición Externa (EE) } \\
\hline 13 Adeudados Externos / Activos & - & -0.6866 & -0.4476 & 0.0033 & 0.5134 & 0.8424 & LogLogistic \\
\hline
\end{tabular}

(-) mayor indice negativo, menor estabilidad, se le asigna el puntaje de 6 si sobrepasa el percentil 90.

\subsection{Estimación del Índice de Estabilidad Bancaria (IEB)}

Luego de haber obtenido los percentiles, se procedió a estimar el IEB para cada uno de los cuatro bancos analizados, y luego para el agregado o total de ellos, los resultados para el total se presentan en los gráficos 3 y 4. De manera general el IEB tanto en niveles como en diferencia nos señala que los 4 bancos grandes están fuera del nivel más riesgoso (6); en segundo lugar, el IEB en niveles muestra una tendencia descendente en el riesgo; y en tercer lugar, el IEB en diferencia presenta una evolución relativamente más estable entre las calificaciones de 3 y 4, rango catalogado como una situación de riesgo moderado. Esto resultados obtenidos son consistentes y similares a los obtenidos en el trabajo publicado por el Banco Central de Reserva del Perú (Espino, 2012) para el periodo 2000 - 2011.

El hecho que el IEB en diferencia se mantenga en un rango de riesgo moderado o expectante, implica que uno o algunos de los indicadores que la conforman pueden estar presentando situaciones de alto riesgo y que a su vez se cuenten con otros indicadores que están siendo bien controlados. Esto hace que analicemos la evolución de cada indicador, lo que no permite extendernos al detalle para cada uno de ellos, por lo que solo haremos referencia a uno de los indicadores principales como es el ratio de cartera morosa / colocaciones brutas. Como se presenta en el gráfico 5, los valores normalizados de este último ratio muestran una tendencia creciente en niveles, indicando una tasa de morosidad en ascenso, exponiendo la estabilidad bancaria a un alto riesgo.

Gráfico 3. Índice de Estabilidad Bancaria (IEB) en Niveles.

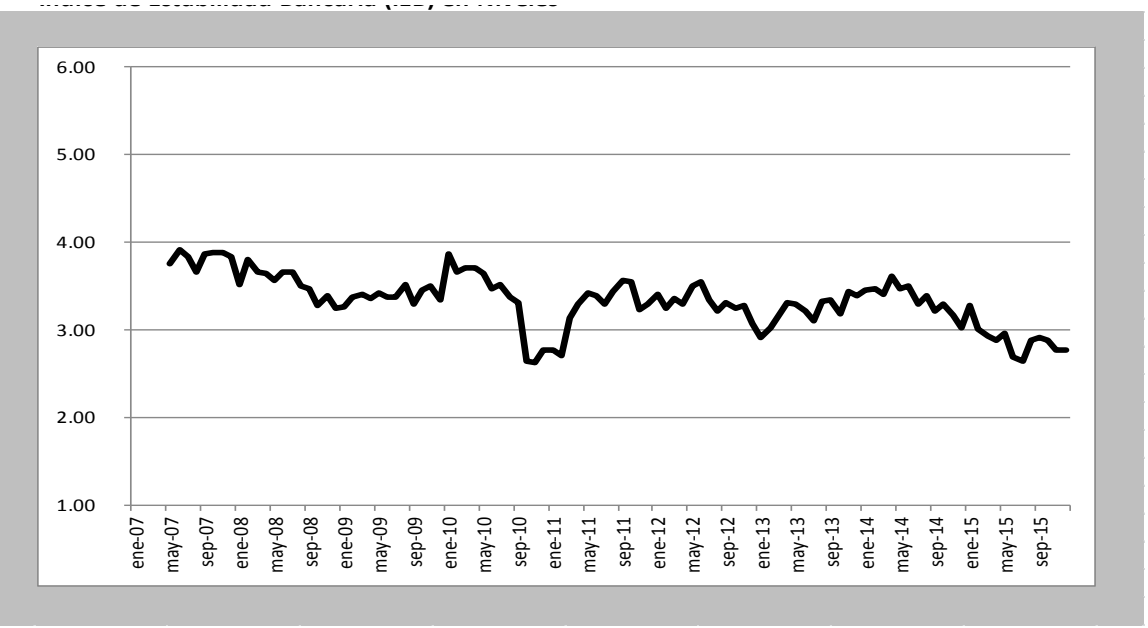


Gráfico 4. Índice de Estabilidad Bancaria (IEB) en Diferencia.

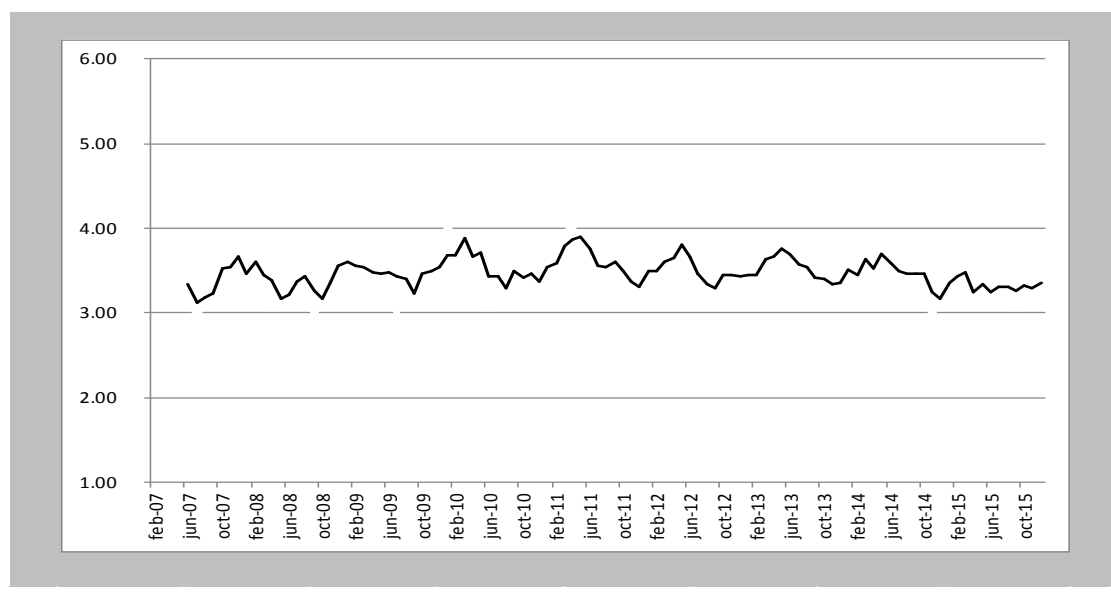

Gráfico 5. Índice Ratio Cartera Morosa / Colocaciones Brutas (en Niveles).

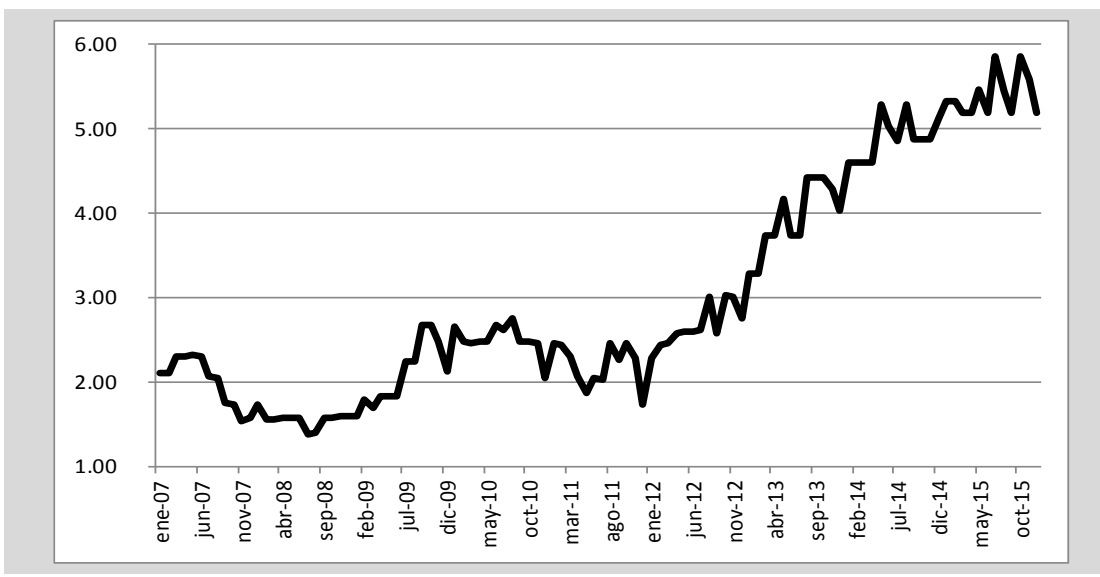

Gráfico 6. Índice Ratio Cartera Morosa / Colocaciones Brutas (en Diferencia).

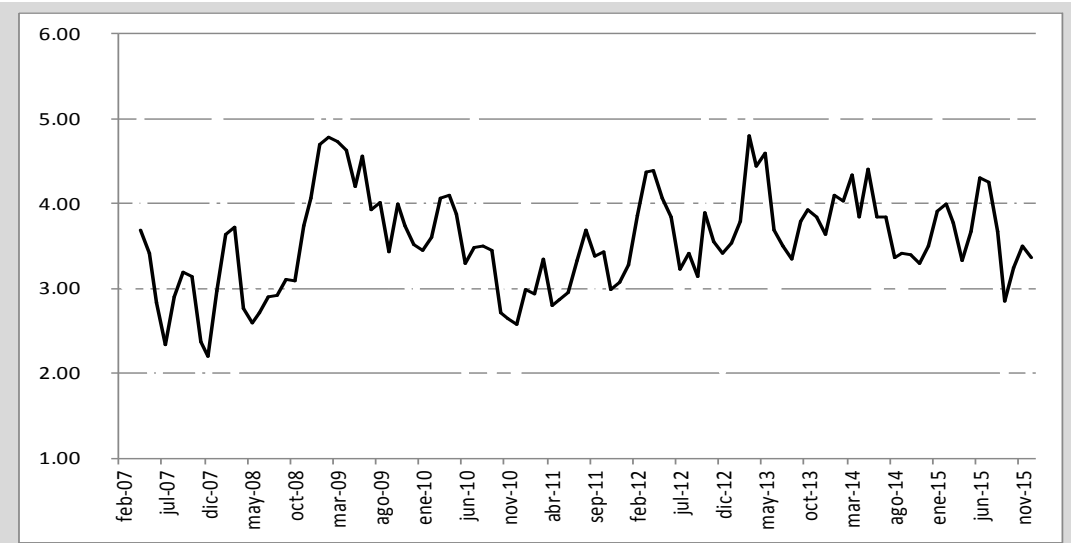

medidas para aumentar la liquidez en el sistema financiero y permitir la normal fluidez del crédito, tal como se puede constatar en los informes de política monetaria y los reportes de estabilidad financiera que publica de manera periódica el ente emisor del país, es decir el rol de la Banca Central fue decisivo en contribuir a la estabilidad bancaria en los años 2008 y 2009.

Posteriormente, los años 2011 al 2013 y hacia adelante, nuevamente la desaceleración de la economía mundial (China, Europa y los Estados Unidos de Norte América), así como la política de subir la tasa de interés en los Estados Unidos por parte de la Reserva Federal de ese país, está complicando el crecimiento económico mundial, la generación de rentas y afectar el flujo de cobranzas de los bancos, registrándose tasas de morosidad cada vez más altas, alcanzando en promedio y cercano al $3 \%$ para los cuatro bancos analizados al final del 2015; nuevamente en este caso el Banco Central de Reserva maneja la política monetaria a garantizar la liquidez en el sistema financiero y tasas de interés bajas.

Finalmente, la presencia del sistema de regulación a que se rigen los bancos, en administrar un adecuado monto de capital libre de riesgo de acuerdo al marco regulador internacional para bancos "Basilea III" $\left({ }^{2}\right)$, aunado a conseguir buenos indicadores de liquidez (fondos disponibles / depósitos totales) y a una buena gestión del riesgo de la cartera en moneda extranjera al reducir la exposición de los créditos en dólares a la volatilidad y alza del tipo de cambio, todo ello está permitiendo compensar el alza de la tasa de morosidad bancaria ya mencionada, y

Efectivamente, si tenemos en cuenta el contexto, de la mano con la crisis internacional del 2008 y la caída de la demanda internacional de ese año complicó un poco la cadena de pagos reflejado en buena parte con tasas de morosidad en ascenso en el sistema bancario. Desde el último trimestre del 2008, los créditos bancarios a las empresas comenzaron a descender, esto debido a una menor demanda de los prestatarios por la acumulación de inventarios, propio del menor dinamismo de la economía mundial; pero desde setiembre de ese año, ya el Banco Central de Reserva del Perú había adelantado mantener un riesgo moderado de la estabilidad bancaria.

En los gráficos 7 al 10 se presentan los resultados obtenidos del IEB para cada uno de los 4 grandes bancos que han sido objeto de la investigación, datos cuya fuente son las estadísticas de la SBS, los mismos que fueron procesados en el software econométrico STATA 14,

\footnotetext{
2"Basilea III" es un conjunto integral de reformas elaborado por el Comité de Supervisión Bancaria de Basilea (G-10) para fortalecer la regulación, supervisión y gestión de riesgos del sector bancario, con el propósito de mejorar la capacidad del sector bancario para afrontar perturbaciones ocasionadas por tensiones financieras o económicas de cualquier tipo; mejorar la gestión de riesgos y el bueno gobierno en los bancos; y reforzar la transparencia y la divulgación de información de los bancos.
} 


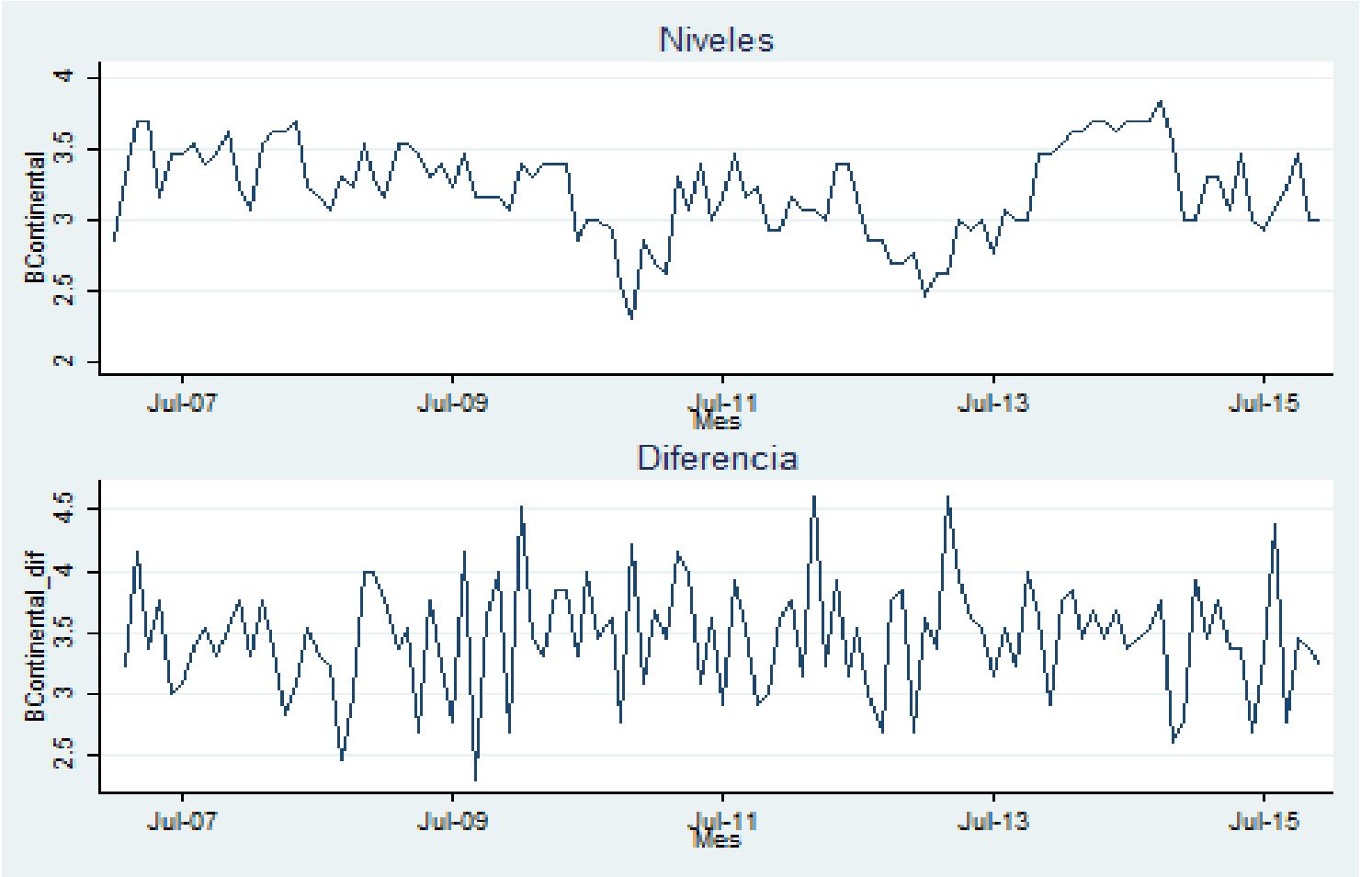

Gráfico 7. Indice de Estabilidad Bancaria BBVA en niveles (superior) y en diferencias (inferior).

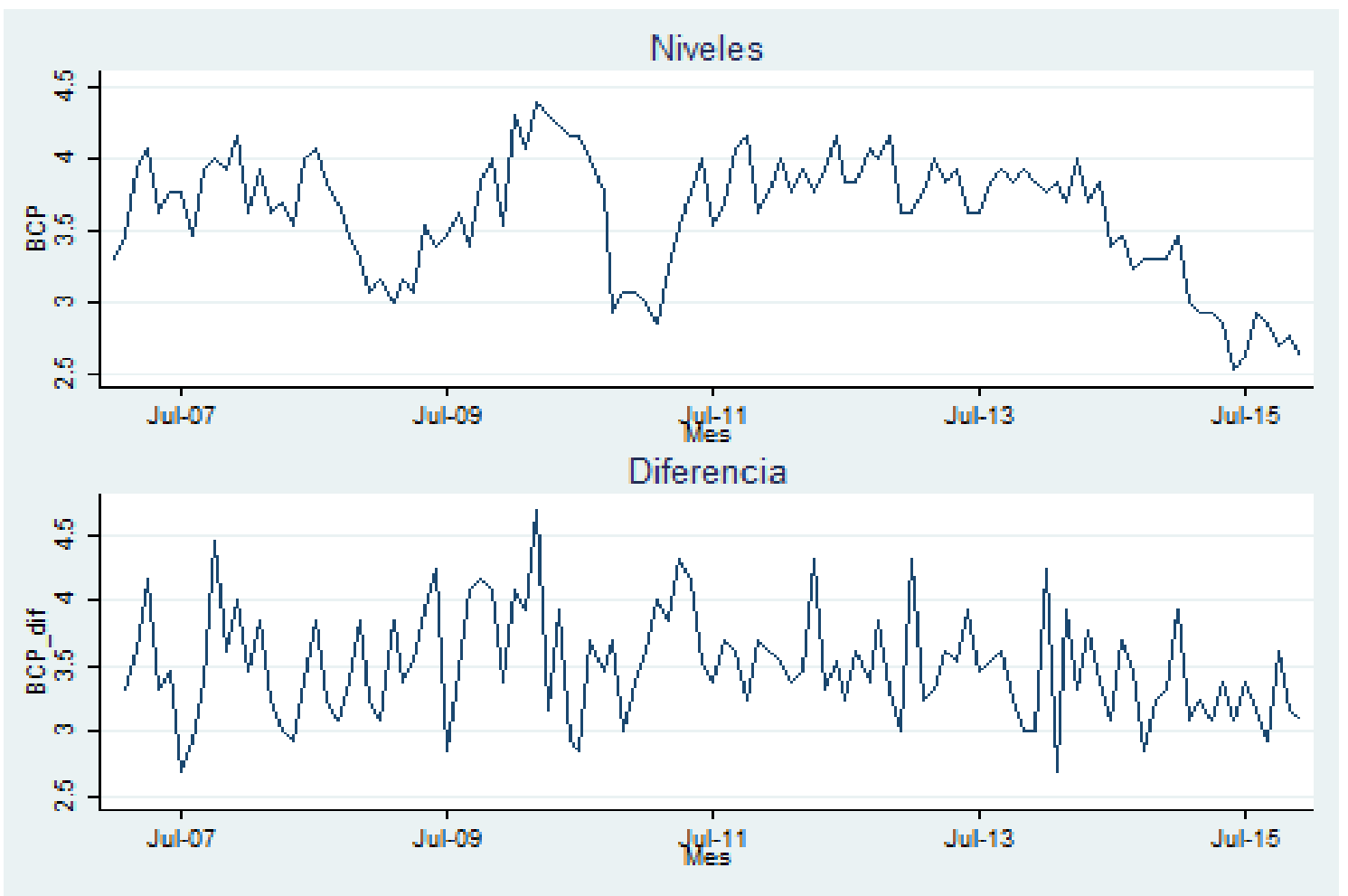

Gráfico 8. Índice de Estabilidad Bancaria del Banco de Crédito del Perú. 


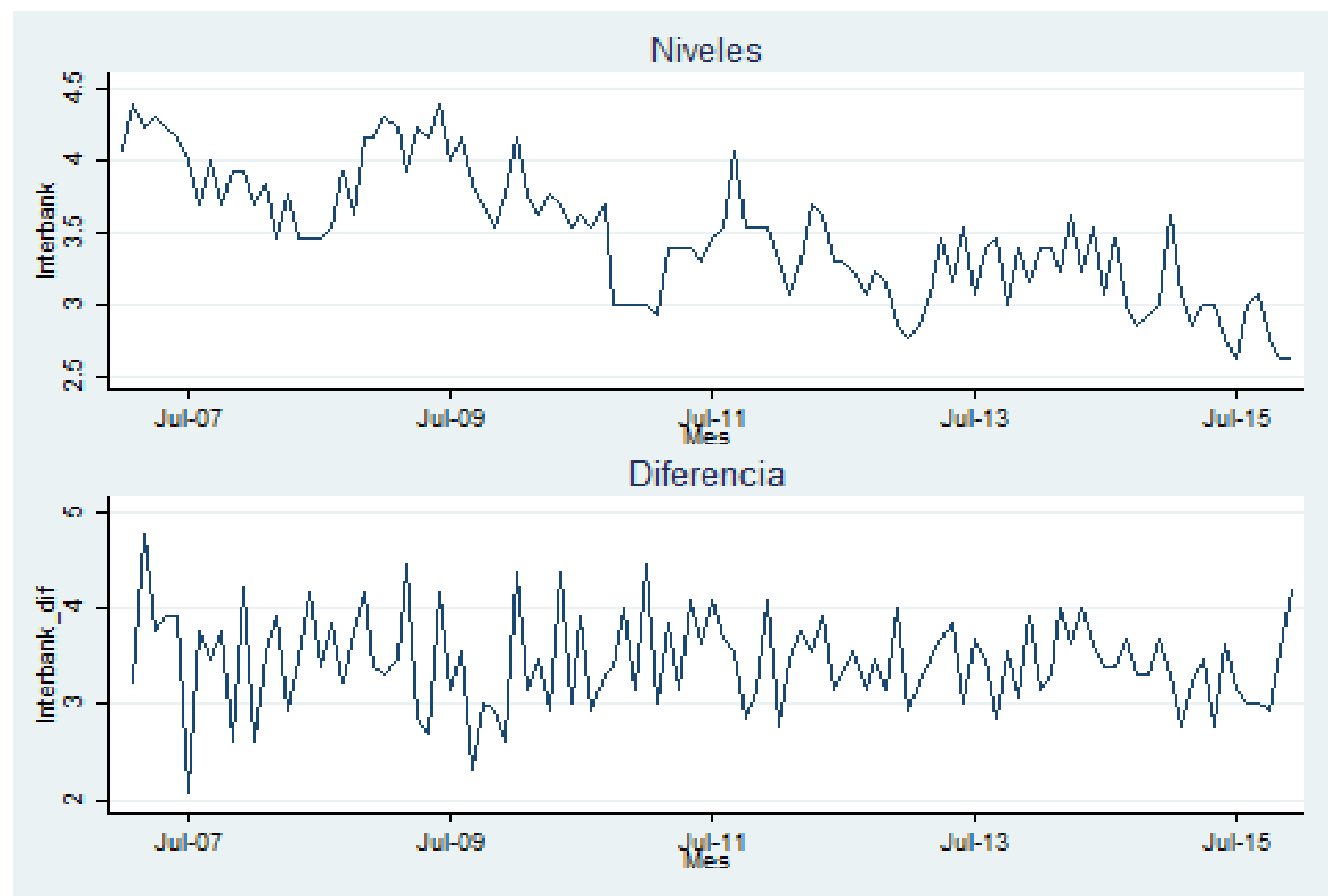

Gráfico 9. Índice de Estabilidad Bancaria del Banco Interbank

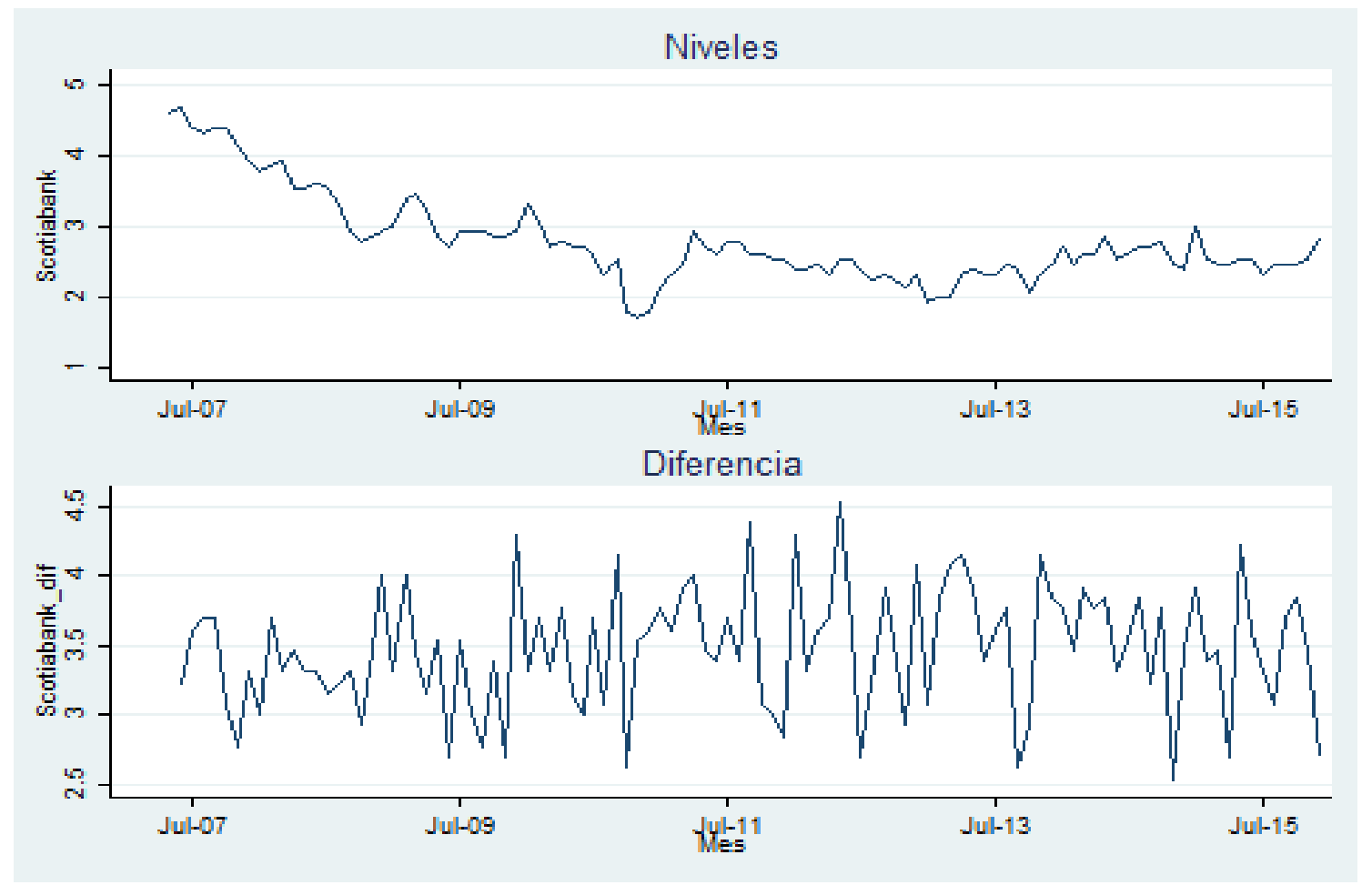

Gráfico 10. Índice de Estabilidad Bancaria del Banco de Scotiabank 


\section{Conclusiones}

Una primera conclusión es la demostración de los resultados del riesgo financiero de los cuatro principales bancos que concentran más del $85 \%$ de las colocaciones, los que han logrado mantenerse en un nivel de riesgo moderado, de acuerdo a los criterios de calificación presentados en esta investigación, tras haber hecho frente a la severa crisis económica internacional del 2008 y de la recesión económica desde el 2011 hasta el 2015, incluso hasta la actualidad en que se suscribe este documento.

Una segunda conclusión es que en el largo plazo, la tendencia del sistema bancario es a reducir levemente el índice de estabilidad bancaria y con ello ofreciendo una estabilidad del sistema bancario en nuestro país, lo que implica que el sistema bancario se encuentra en una buena posición sólida a prueba de shocks externos muy importantes.

Destacar de manera adicional, que si bien la gestión financiera de los bancos están mostrando indicadores relativamente estables, esto viene siendo posible a la presencia de un rol más cauteloso del Banco Central de Reserva, de la entidad supervisora nacional y de los arreglos institucionales en materia de regulación bancaria.

\section{Literatura citada}

Borio, C., y Drehmann, M. 2009. «Towards an operational framework for financial stability:'fuzzy'measurement and its consequences». JOUR. BIS Working Paper. http:// www.bis.org/publ/work284.pdf.

De Jonghe, O. 2010. «Back to the basics in banking? A micro-analysis of banking system stability». JOUR. Journal of Financial Intermediation 19 (3): 387-417. doi:http://dx.doi.org/10.1016/j.jfi.2009.04.001.

Delgado, Carlos, y Yucaris García. 2013. «Un índice de estabilidad bancaria para la República Dominicana: una aproximación cuantitativa de estabilidad financiera». http://www.bancentral.gov.do/bibliotecap/pdf/ganadores/ segundo.pdf.

Demirgüç-Kunt, Asli, y Vojislav Maksimovic. 1998. «Law, finance, and firm growth». JOUR. The Journal of Finance 53 (6). Wiley Online Library: 2107-37. http://siteresources.worldbank.org/DEC/Resources/ lawfinanceandfirmgrowth.pdf.

Espino, F. 2012. «Un índice de estabilidad bancaria para Perú». JOUR. BCRP Serie de Documentos de trabajo DT. http://200.37.165.6/docs/Publicaciones/Documentos-deTrabajo/2012/documento-de-trabajo-15-2012.pdf.

Estrada, D., y Morales, M. 2009. «Índice de Estabilidad Financiera para Colombia». JOUR. Reporte de Estabilidad Financiera. http://www.banrep.gov.co/docum/Lectura finanzas/pdf/marzo_indice.pdf.

Illing, M. y Ying, L. 2003. An index of financial stress for Canada. BOOK. Bank of Canada. http://www. bankofcanada.ca/wp-content/uploads/2010/02/wp03-14. pdf.
IMF. 2006. «Financial Soundness Indicators: Compilation Guide». International Monetary Fund. https://www.imf. org/external/pubs/ft/fsi/guide/2006/pdf/fsiFT.pdf.

Jahn, N. y Kick, T. s. f. «Determinants of banking system stability: A macro-prudential analysis». JOUR. http://www.bis.org/bcbs/events/bhbibe/jahn.pdf.

Jokipii, Terhi, y Pierre Monnin. 2013. «The impact of banking sector stability on the real economy». JOUR. Journal of International Money and Finance 32 (febrero): 1-16. doi:http://dx.doi.org/10.1016/j. jimonfin.2012.02.008.

King, R. G, y Levine, R. 1993a. «Finance, entrepreneurship and growth». JOUR. Journal of Monetary economics 32 (3). Elsevier: 513-42.

1993b. «Finance and growth: Schumpeter might be right». JOUR. The quarterly journal of economics. JSTOR, 717-37. http://qje.oxfordjournals. org/content/108/3/717.abstract.

Levine, R. 1997. «Financial development and economic growth: views and agenda». JOUR. Journal of economic literature 35 (2). JSTOR: 688-726. http://pascal.iseg.utl. pt/ aafonso/eif/pdf/Levine.pdf.

. 1998. «The legal environment, banks, and longrun economic growth». JOUR. Journal of money, credit and banking. JSTOR, 596-613. http://www.jstor.org/ stable/2601259?seq=1\#page_scan_tab_contents.

Levine, R., Loayza, N. y Beck, T. 2000. «Financial intermediation and growth: Causality and causes». JOUR. Journal of monetary Economics 46 (1). Elsevier: 31-77. http://faculty.haas.berkeley.edu/ross_levine/ papers/2000_JME_Fin Int Growth Causality.pdf.

Ortiz, Vidal-Abarca, A. y Ugarte, A. 2015. «Introducing a New Early Warning System Indicator (EWSI) of banking crises». RPRT. https://www.bbvaresearch.com/ wp-content/uploads/2015/01/WP_EWS-SystemVersionSep2014_i.pdf.

Puddu, S. 2008. «Optimal Weights and Stress Banking Indexes». JOUR. Lausanne: HEC-Université de Lausanne. Citeseer. https://editorialexpress.com/cgi-bin/conference/ download.cgi?db_name=simposio2008\&paper_id=283.

Rajan, Raghuram G, y Zingales, L. 1998. «Financial Dependence and Growth». JOUR. The American Economic Review 88 (3). American Economic Association: 559-86. http://www.jstor.org/stable/116849.

\section{Anexo}

Obtención de los Percentiles para los 13 indicadores 

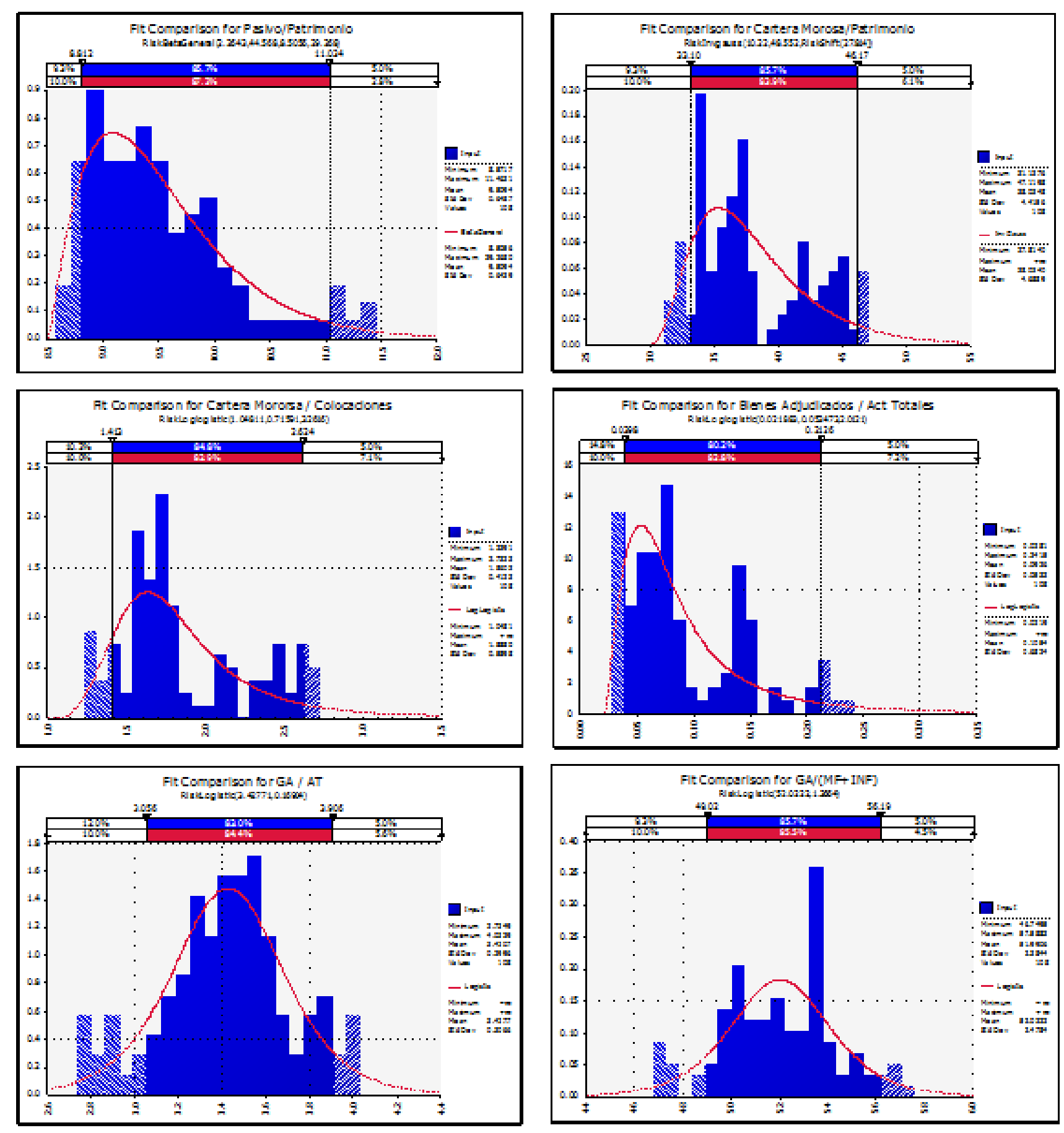

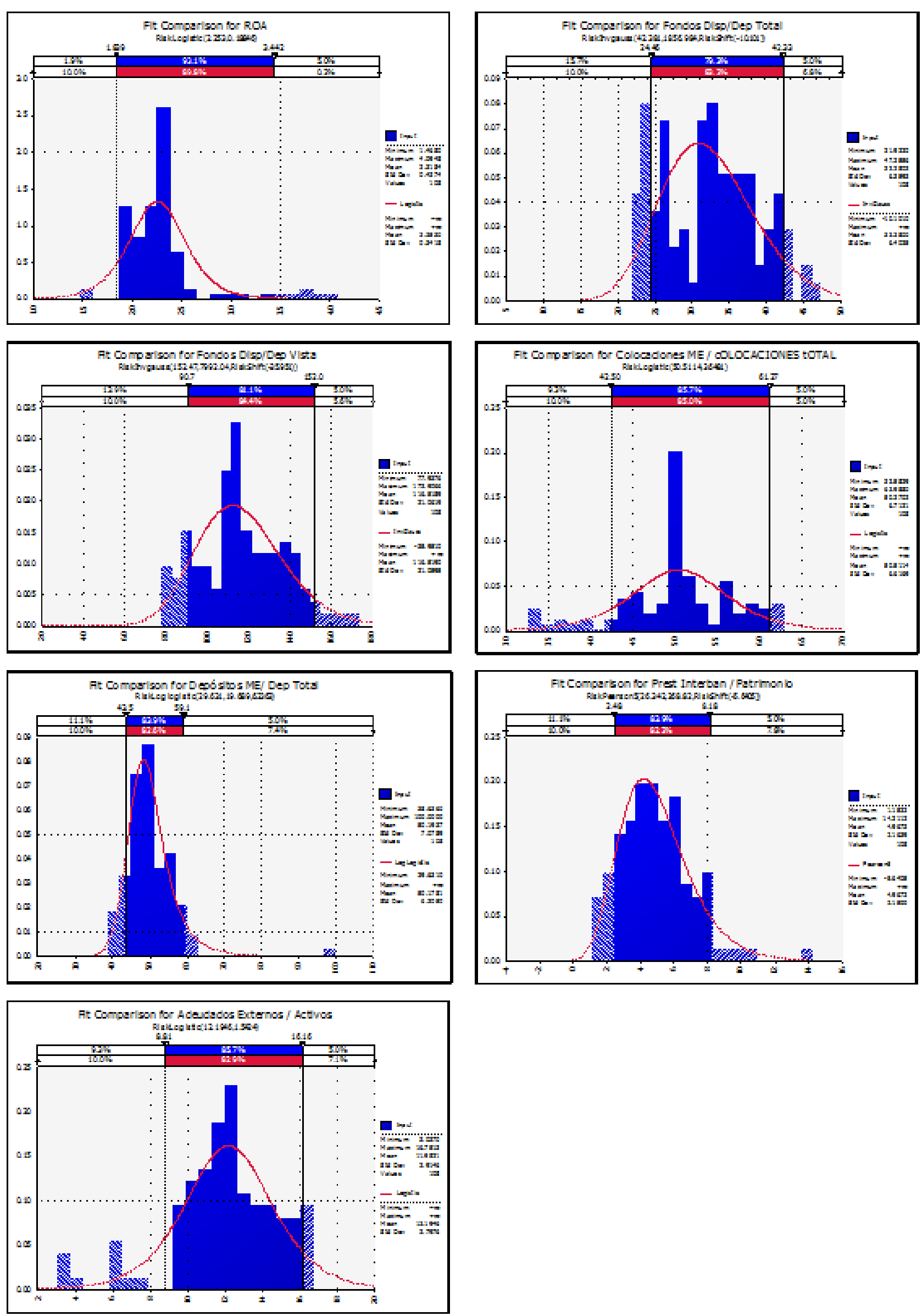